\title{
La recherche qualitative en pédagogie médicale : histoire, pratique et légitimité
}

\section{Qualitative research in medical education: history, practice and legitimacy}

\author{
Thierry Pelaccia ${ }^{1,2}$ et Pierre Paillé ${ }^{3,4}$
}

1 Service d'aide médicale urgente (SAMU 67) et Centre d'enseignement des soins d'urgence (CESU 67), Hôpitaux universitaires de Strasbourg, Strasbourg, France

2 Laboratoire de recherche en pédagogie des sciences de la santé, Faculté de médecine de Strasbourg, Strasbourg, France

3 Faculté d'éducation, Université de Sherbrooke, Québec, Canada

4 Groupe de recherche "Souffrance et médecine », Centre hospitalier universitaire de Montréal (CHUM), Québec, Canada

Manuscrit reçu le $1^{\text {er }}$ février 2011; commentaires éditoriaux formulés aux auteurs le 19 mai 2011; accepté pour publication le 2 juin 2011

\section{Mots clés :}

Méthodes qualitatives ; positivisme ; ethnographie ; théorie ancrée ; étude de cas ; scientificité

\section{Keywords:}

Qualitative methods; positivism; ethnography; grounded theory;

\section{Messages clés}

- Un nombre croissant de chercheurs dans le champ de l'éducation médicale est partisan de la doctrine du «best-fit», consistant à associer à une question de recherche le paradigme et les méthodes les plus appropriés.

- Malgré une tradition ancienne de recherche qualitative initiée dans le cadre du courant ethnographique et l'essor de ces approches depuis les années quatre-vingt, la «bataille» visant à en démontrer la légitimité dans le champ de la recherche en éducation médicale n'est pas encore gagnée.

- Dans un environnement marqué par le gold standard de l'étude randomisée et contrôlée, certains considèrent les travaux de recherche qualitative comme de simples études descriptives ne remplissant pas les critères de scientificité de la recherche.

- Concernant la pratique de la recherche qualitative dans le champ de l'éducation médicale :

- Elle s'inscrit essentiellement dans le cadre du paradigme postpositiviste, lequel en fondé en bonne partie sur une ontologie réaliste et une épistémologie objectiviste ;

- L'ethnographie, la théorie ancrée et l'étude de cas sont les stratégies les plus couramment utilisées ;

- Les approches qualitatives sont souvent appréhendées comme de simples méthodes de recueil et d'analyse des données, s'inscrivant dans une forme de pratique «aparadigmatique » de la recherche;

- Les principes méthodologiques sont fréquemment appliqués partiellement et sélectivement;

- Les critères de rigueur utilisés relèvent de la tradition postpositiviste de la recherche scientifique;

- Les entretiens semi-dirigés sont le mode privilégié de collecte des données.

\section{Key messages}

- A growing number of researchers in medical education believe in the "best-fit" doctrine, which consists in choosing the best paradigm and methods in regard to the research question. 


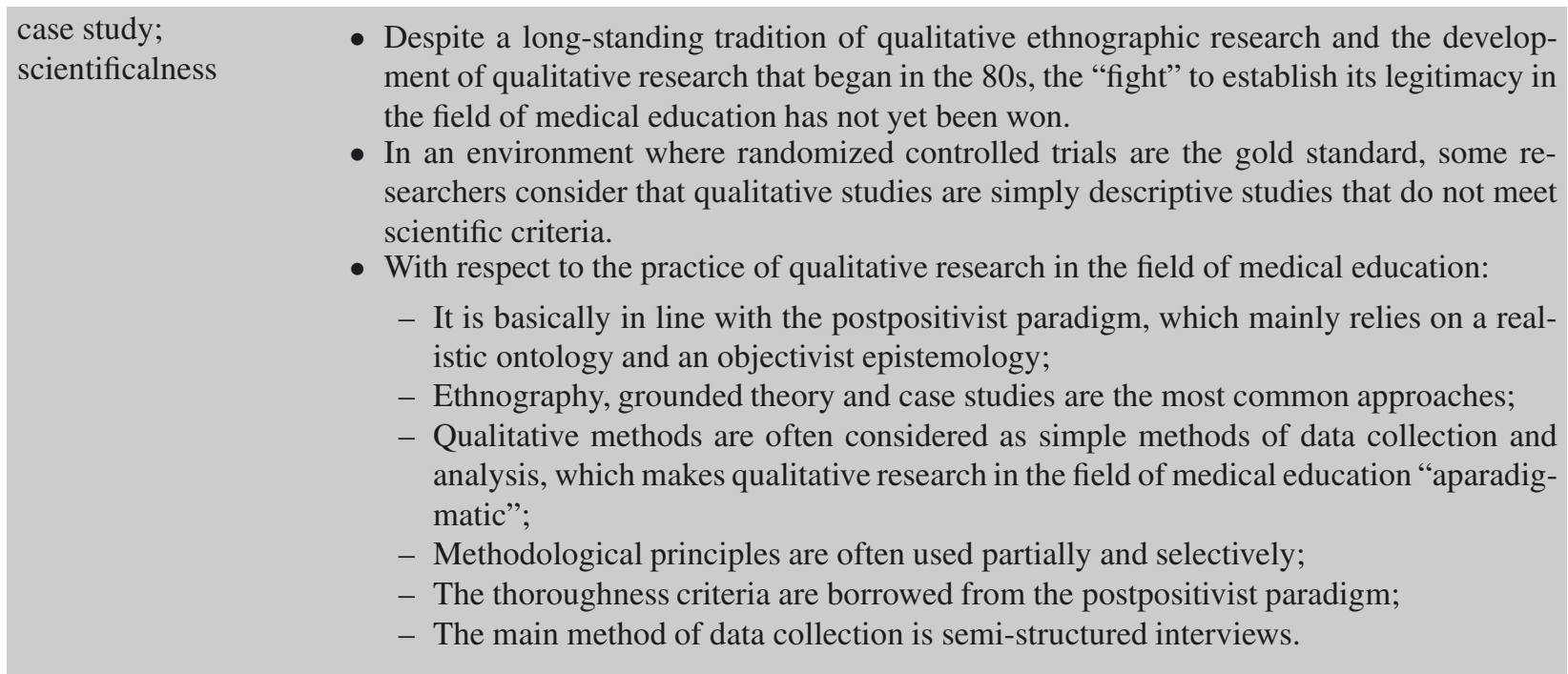

\section{Introduction}

En matière de recherche, méthodes qualitatives et méthodes quantitatives font fréquemment l'objet d'une opposition binaire. Cet «affrontement» est patent dans le discours de la pédagogie médicale ${ }^{[1]}$ (dans cet article, les termes «pédagogie médicale» et « éducation médicale » seront utilisés indifféremment pour désigner le champ auquel nous nous intéressons). Pourtant, cette vision antagoniste et conflictuelle a fait l'objet de mises en garde quant à sa pertinence, tant dans le domaine de l'éducation $^{[2,3]}$, que dans celui de la pédagogie médicale $^{[1,4]}$. Un nombre croissant de chercheurs est ainsi partisan de la doctrine du «best fit », consistant à associer à une question de recherche le paradigme et les méthodes les plus appropriés ${ }^{[1]}$. Cette vision « réconciliatrice »- prônée dès le début des années quatre-vingt-dix ${ }^{[5]}$, réaffirmée à l'aube des années deux-mille ${ }^{[6]}$ et récemment réitérée par Lingard et Kennedy ${ }^{[1]}$ et par Pelaccia et Paillé ${ }^{[4]}$ - tend à masquer la persistance d'un vif débat quant à la place des approches qualitatives en recherche en éducation, notamment médicale. Norman le qualifiait de « champ de bataille » et, de façon plus préoccupante, estimait qu'il n'y avait pas de solution en vue ${ }^{[6]}$.
Colliver partage cette vision et considère que ce débat traduit un penchant de la communauté des chercheurs en éducation pour l'autodestruction ${ }^{[7]}$.

Afin de contribuer à cette discussion, nous nous intéresserons dans un premier temps à la façon dont la recherche qualitative est définie dans le domaine de la pédagogie médicale. Nous en retracerons ensuite l'émergence et le développement. Parmi les différents courants, nous identifierons ceux qui font l'objet d'un intérêt particulier de la part des chercheurs du champ, tenterons d'en expliquer la raison et discuterons de la façon dont ils sont appréhendés. Il s'agira en particulier de déterminer s'ils s'inscrivent en continuité ou en rupture avec les pratiques des sciences humaines et sociales. Dans cette perspective, nous mettrons en évidence les racines épistémologiques et ontologiques de la pratique de la recherche qualitative en éducation médicale.

En avant-propos, il convient de souligner que notre objectif n'a bien entendu pas été de réaliser une revue extensive de la littérature afin de prendre connaissance de l'ensemble des travaux de recherche qualitative publiés dans les journaux de pédagogie médicale. Il s'agit plutôt d'essayer d'identifier des repères et des tendances quant à la 
venue de ces approches, à leur place et à la façon dont elles sont pratiquées au sein du champ.

Sur le plan méthodologique, plusieurs ressources ont servi de point de départ à l'exploration de la littérature scientifique en lien avec la thématique étudiée :

- Les articles traitant des approches qualitatives en éducation médicale, identifiés lors d'une recherche dans la base de données Medline, à partir des termes MeSH «Education, Medical» et "Qualitative Research», liés par l'opérateur booléen «AND».

- Les articles traitant des approches qualitatives en éducation médicale, obtenus lors d'une recherche manuelle sur le site de la revue Pédagogie Médicale.

- Le manuel de recherche en éducation médicale publié par Norman, van der Vleuten et Newble ${ }^{[8]}$.

D'autres ressources ont ensuite été incluses à partir des références citées dans les documents utilisés initialement.

\section{Définition de la recherche qualitative en éducation médicale}

Dans un ouvrage de référence sur la recherche en éducation médicale, on peut lire que les chercheurs qualitatifs conçoivent la connaissance comme le fruit d'une construction par l'homme ${ }^{[8]}$. Ils tentent d'appréhender la complexité des interactions sociales, des expériences des individus et des processus sociaux, à travers les questions du «comment?» et du «quoi ?» ${ }^{[1]}$. Dans le domaine de l'éducation médicale, il s'agirait, par exemple, de s'intéresser aux relations médecins-malades, à la prise de décision ou encore, aux questions identitaires.

D'un point de vue méthodologique, Lingard et Kennedy soulignent qu'il est nécessaire que ces études prennent place dans un environnement naturel, où le chercheur peut observer et interagir avec les individus qui vivent le phénomène sur lequel il porte son attention ${ }^{[1]}$. Il ne s'agit pas de viser la généralisabilité mais de valoriser «la compréhension située et la construction de théories » ${ }^{[1]}$. Dans ce cadre, l'observation, les entretiens et les ressources documentaires constituent les principaux outils de recueil des données ${ }^{[1,8]}$. Quant à l'analyse, elle repose sur le principe du codage et relève d'une approche interprétative.

Dans les manuels de référence en éducation médicale, les approches qualitatives sont donc appréhendées comme une démarche globale de recherche, incluant ses aspects épistémologiques. Nous considérons que cette posture est comparable à celle décrite en sciences humaines et sociales, par exemple par Denzin et Lincoln ${ }^{[9]}$. Il est toutefois légitime de s'interroger sur le fait que cette conception de la recherche qualitative - présentée par des chercheurs issus du domaine des sciences humaines et sociales et travaillant au sein de départements et de centres de pédagogie médicale - pourrait relever d'une intention prescriptive et idéalisante, qui n'est pas forcément le reflet de la façon dont les approches qualitatives sont réellement pratiquées en recherche en pédagogie médicale. C'est précisément à cette pratique et à son histoire que nous allons nous intéresser dans les prochaines sections.

\section{Comment la recherche qualitative et ses principaux courants sont-ils apparus et se sont-ils développés en éducation médicale?}

\section{Une tradition ancienne}

Il existe étonnamment une tradition ancienne de recherche qualitative en éducation médicale ${ }^{[10]}$. Les premiers travaux marquants ont été réalisés par des sociologues américains à la fin des années cinquante, dans le cadre du courant ethnographique. Merton, Reader et Kendall se sont ainsi intéressés à la socialisation des étudiants en médecine ${ }^{[11]}$. Quelques années plus tard, Becker, Geer, Hughes et Strauss ont étudié la culture étudiante et la façon dont les futurs médecins vivent leurs expériences 
d'apprentissage, construisent leur identité professionnelle et apprennent leur métier ${ }^{[12]}$. Forquin considère ce travail comme "l'une des œuvres les plus représentatives de la tradition de recherche de l'école de Chicago », en raison de l'utilisation rigoureuse et riche de la méthode d'observation ${ }^{[13]}$. Au cours des décennies suivantes, d'autres études ont été ponctuellement réalisées dans ce champ par des sociologues, des anthropologues et des éducationnistes. Il s'agit des travaux de Bloom dans les années soixante-dix, d'Atkinson et de Weinholtz dans les années quatre-vingt et de Sinclair dans les années quatre-vingt-dix ${ }^{[8,10,13,14]}$.

La porte d'entrée de la recherche qualitative en éducation médicale se caractérise donc par le fait :

- qu'elle a été ouverte par des sociologues œuvrant au sein de départements de sociologie ${ }^{[8]}$. L'intitulé de l'introduction de l'ouvrage de Merton et al. - «Some preliminaries to a sociology of medical education »- laisse d'ailleurs peu de doutes sur le fait que ce travail vise avant tout la communauté des sociologues ${ }^{[11]}$;

- qu'elle est marquée par l'approche ethnographique $^{[14]}$ et que certains travaux s'inscrivent dans la lignée de ceux de l'école de Chicago ${ }^{[13]}$;

- qu'elle s'est tour à tour refermée puis rouverte, comme en atteste le caractère très ponctuel de ces études.

En apparence, il existe donc une tradition ancienne de recherche qualitative dans le champ de l'éducation médicale. Plus exactement, il s'agit d'une démarche entreprise par des sociologues, des anthropologues et des éducationnistes œuvrant au sein de leurs départements respectifs, qui ont choisi comme terrain d'étude le lieu de formation des étudiants en médecine ${ }^{[1]}$. Ces travaux ne sont donc pas stricto sensu ancrés dans le champ disciplinaire de la pédagogie médicale. Ils n'ont d'ailleurs pas eu d'influence significative sur les pratiques méthodologiques des acteurs du champ ${ }^{[8]}$, qui se sont longtemps montrés hostiles à toute forme de recherche s'éloignant du paradigme positiviste.
L'essor de la recherche qualitative en éducation médicale

Du début du $\mathrm{XX}^{\mathrm{e}}$ siècle jusqu'aux années quatrevingt, le paradigme positiviste (ou post-positiviste, à savoir la version aménagée du positivisme qui émerge progressivement au milieu du $\mathrm{XX}^{\mathrm{e}}$ siècle) et sa démarche expérimentale ont été les «standards » de la recherche en éducation médicale (tableau I) ${ }^{[8]}$. Comme le soulignent à ce sujet Bunniss et Kelly, « les médecins, les éducateurs médicaux et les chercheurs en éducation médicale ont des perspectives et des expériences de recherche qui ont été marquées par une philosophie de la connaissance scientifique associée à la tradition positiviste de la médecine » ${ }^{[16]}$. Toutefois, depuis le début des années quatre-vingt, les chercheurs ont à plusieurs reprises affirmé et argumenté l'apport potentiel des approches qualitatives (notamment ethnographiques) au domaine de l'éducation médicale ${ }^{[1,8]}$. «L'idée d'une recherche qualitative en éducation médicale » ${ }^{[17]}$ s'est en particulier accentuée à la fin des années quatre-vingt, avec la publication - dans les journaux et les congrès du champ - de tribunes sur son intérêt, ses fondements et ses principes ${ }^{[8]}$. La popularité de ces approches s'est encore accrue au début des années deux-mille, notamment grâce au développement des logiciels de traitement des données ${ }^{[8,18]}$. Les méthodologies de recherche qualitative semblent donc globalement acceptées dans le champ de l'éducation médicale ${ }^{[8,19]}$, comme en témoigne la possibilité croissante d'y publier des articles basés sur ces approches, qui étaient auparavant d'emblée rejetés en raison de leur caractère «non scientifique » ou « anecdotique ${ }^{[10]}$.

De nombreux travaux de recherche qualitative ont depuis été réalisés dans ce domaine ${ }^{[8,19,20]}$. Certaines thématiques ont plus particulièrement été appréhendées sous cet angle, probablement en raison de leur complexité. Il s'agit de l'enseignement et de l'apprentissage en milieu hospitalier, ambulatoire ou dans le cadre des dispositifs d'apprentissage par problème, de l'influence des modèles de rôle sur le choix des spécialités, de l'évaluation de programmes, des questions relatives au développement 
Tableau I. Comparaison du positivisme et du constructivisme d'un point de vue ontologique, épistémologique, téléologique et méthodologique.

\begin{tabular}{ccc} 
& Positivisme & Constructivisme \\
\hline $\begin{array}{c}\text { Ontologie } \\
\text { (nature de la réalité) }\end{array}$ & $\begin{array}{c}\text { La réalité existe } \\
\text { indépendamment du sujet } \\
\text { (réaliste) }\end{array}$ & $\begin{array}{c}\text { Les réalités sont multiples et font } \\
\text { l'objet d'une construction } \\
\text { spécifique et locale (relativiste) }\end{array}$ \\
\hline $\begin{array}{c}\text { Épistémologie } \\
\text { (nature de la }\end{array}$ & $\begin{array}{c}\text { La connaissance est extérieure } \\
\text { connaissance) }\end{array}$ & $\begin{array}{c}\text { La connaissance est le fruit d'une } \\
\text { (co)construction par l'individu et ce qui }\end{array}$ \\
\hline Téléologie & est vrai (dualiste, objectiviste) & est observé est créé ([inter]subjectiviste) \\
\hline (finalité) & explicative & compréhensive \\
Méthode & (expliquer la réalité) & (comprendre une réalité) \\
\hline & quantitative expérimentale & qualitative et quantitative
\end{tabular}

Le post-posivitisme se distingue du positivisme en considérant que la réalité ne peut être saisie que de façon incertaine et partielle (réalisme critique) et que la vérité ne peut être qu'approchée (dualisme modifié).

curriculaire, au professionnalisation, au bien-être des médecins et aux relations au sein des équipes de $\operatorname{soin}^{[8,19,20]}$.

\section{Le développement des différents courants} de la recherche qualitative en éducation médicale

L'ethnographie, la théorie ancrée et l'étude de cas sont les stratégies les plus couramment utilisées par les chercheurs en éducation médicale ${ }^{[8,19]}$.

\section{Le courant ethnographique}

Comme nous l'indiquions précédemment, le courant ethnographique a véritablement marqué l'émergence des approches qualitatives en éducation médicale. Depuis un demi-siècle, plusieurs travaux ont été réalisés dans ce cadre. Ils ont porté sur la culture étudiante au sein des facultés de médecine, sur le curriculum «caché » et sur les conséquences involontaires des réformes dans ces institutions ${ }^{[14]}$. Ces études ont toutefois essentiellement été entreprises par des chercheurs travaillant au sein de départements de sciences humaines et sociales ${ }^{[1,14]}$.
Nous identifions plusieurs obstacles au développement d'une pratique de recherche ethnographique propre au champ de l'éducation médicale :

- Que l'on définisse l'objet d'étude de l'ethnographie en lien avec les connaissances de nature culturelle, les interactions sociales ou l'analyse des sociétés ${ }^{[21]}$, ces thématiques sont fondamentalement éloignées des préoccupations des éducateurs médicaux, marquées par la dimension pédagogique de la formation des futurs médecins ${ }^{[22-25]}$.

- La pratique ethnographique exige de la part du chercheur une immersion directe et prolongée dans le milieu étudié ${ }^{[1,8,14,21]}$. Comme le soulignent à ce sujet Atkinson et Pugsley, «cette approche ne se prête pas aux travaux à court terme » ${ }^{[14]}$. Pour Pope également, «faire de la recherche ethnographique n'est ni rapide, ni simple » ${ }^{[26]}$. Or, les chercheurs en éducation médicale - qui sont fréquemment des praticiens et des enseignants dont l'activité de recherche est secondaire - font souvent preuve d'une « attitude d'impatience par rapport à la collecte des données », qui est peu compatible avec les principes de la recherche ethnographique ${ }^{[20]}$. 
- L'importance de l'observation participante dans la tradition ethnographique renvoie à la possibilité d'une approche subjective de l'objet de recherche $^{[14,27]}$, par rapport à laquelle les éducateurs médicaux sont méfiants.

\section{La théorie ancrée}

La théorie ancrée est le courant qualitatif le plus utilisé en éducation médicale ${ }^{[8,19]}$. Son appropriation par les chercheurs du champ est assez récente $^{[28]}$. Les premiers travaux publiés dans les journaux d'éducation médicale datent ainsi de la fin des années quatre-vingt-dix. Il s'agit de ceux de Burack et al. sur le choix des spécialités, de ceux de Ginsburg et al. sur le développement et l'évaluation du professionnalisme chez les étudiants en médecine et de ceux de Lingard et al. sur la communication interprofessionnelle ${ }^{[1,8,28]}$. Nous identifions plusieurs raisons qui expliquent la popularité de la théorie ancrée dans ce champ :

- La démarche méthodologique proposée par les chercheurs à l'origine de ce courant fait l'objet d'une description approfondie et méthodique $^{[28]}$ - en particulier depuis la publication des travaux de Strauss et Corbin ${ }^{[29]}$-, qui prend souvent la forme de guides ${ }^{[19]}$. Kennedy et Lingard les considèrent comme « une concrétisation et une standardisation salutaires du processus mystique permettant de passer du verbatim aux thèmes » ${ }^{[28]}$.

- La production de connaissances en théorie ancrée s'inscrit dans une forme de rigueur post-positiviste, même si elle s'en éloigne par son design méthodologique, les modalités de l'échantillonnage et la façon d'appréhender les éléments de preuve ${ }^{[19]}$. L'objectif de Glaser et de Strauss était précisément de proposer une démarche aussi rigoureuse qu'en recherche quantitative $^{[19]}$. D'autres auteurs ont toutefois plus récemment prôné une approche constructiviste ou postmoderniste de la théorie ancrée ${ }^{[30]}$.

- Une prise de distance par rapport à la posture historique sur le caractère essentiellement inductif de cette approche a été marquée par Strauss et Corbin $^{[29]}$ et par Charmaz ${ }^{[28]}$. Elle est à l'origine d'une conception de la démarche globale de recherche qui est plus compatible avec les habitudes méthodologiques des chercheurs en éducation médicale.

L'ancrage historique de la théorie ancrée autour du modèle de la «bonne science » constitue donc un élément particulièrement attractif aux yeux des chercheurs en éducation médicale ${ }^{[28]}$.

\section{L'étude de cas}

L'étude de cas est l'une des trois approches qualitatives connaissant le plus de succès auprès des chercheurs en éducation médicale ${ }^{[8]}$. Les premiers travaux marquants inspirés de ce courant ont été publiés dans les journaux d'éducation médicale par Irby, au début des années quatre-vingt-dix ${ }^{[8]}$. Il s'agissait d'appréhender la complexité de l'enseignement au lit du malade. Nous attribuons la popularité de l'étude de cas à plusieurs éléments :

- Ce courant trouve ses origines tant dans la tradition sociologique, que dans celle de la médecine $^{[1]}$. Les médecins avaient donc une connaissance de cette approche avant son appropriation par les chercheurs qualitatifs.

- L'ouvrage de Yin constitue le guide de référence pour les chercheurs en éducation médicale choisissant l'étude de cas ${ }^{[31]}$. Or, l'auteur considère que cette dernière doit respecter les principes de validité de construit, de validité interne, de validité externe et de fiabilité, ce qui l'inscrit dans une forme de rigueur méthodologique familière à cette communauté de chercheurs.

- L'étude de cas vise dans une certaine mesure la généralisation (il s'agit toutefois d'une forme de généralisation analytique et non statistique) ${ }^{[8]}$, pratique en conformité avec les canons positivistes ou post-positivistes. 


\section{Les autres courants de la recherche qualitative}

L'approche phénoménologique (qui vise à appréhender l'essence d'un phénomène social en mettant l'accent sur le vécu et l'expérience subjective des individus) ${ }^{[1,21]}$ demeure globalement à l'écart du répertoire méthodologique des chercheurs en éducation médicale, alors qu'elle est couramment utilisée en sciences infirmières, probablement en raison du fait que cette profession focalise davantage son attention sur le patient et sur son vécu de la maladie ${ }^{[8]}$. Le caractère peu explicite des critères de rigueur et la place importante accordée à la subjectivité ${ }^{[21]}$ qui, comme le souligne Meyor, «fait [...] toute la valeur de l'analyse phénoménologique ${ }^{[32]}$ constituent également à l'évidence des freins au développement de ce courant en éducation médicale. Quant à l'analyse narrative (qui s'intéresse aux récits et aux documents biographiques dans le but de donner du sens à une situation ou à une expérience particulière, en les ancrant notamment dans un contexte sociétal) ${ }^{[1,21]}$ et à l'ethnométhodologie (qui étudie, de l'intérieur, la façon dont les individus construisent leurs pratiques sociales, à partir de leurs interactions et de l'identification des savoirs de sens commun) ${ }^{[21]}$, les chercheurs issus de la tradition positiviste ou post-positiviste peuvent probablement leur adresser des critiques similaires, ce qui en explique l'usage très limité dans le champ de l'éducation médicale.

\section{Un « combat » d'arrière-garde ?}

L'essor des approches qualitatives en recherche en éducation médicale s'est bien entendu accompagné d'un mouvement réactionnel critique, notamment lié à une préoccupation croissante pour scientifiser le champ. Il s'agissait de déterminer dans quelle mesure les pratiques de recherche pouvaient répondre aux exigences de la méthode scientifique et expérimentale ${ }^{[33]}$. Le «gold standard» de l'étude randomisée contrôlée s'est alors transposé de la recherche biomédicale à la recherche en éducation médicale ${ }^{[34]}$, ce qui conduisit Colliver et Verhulst à considérer que « la recherche en éducation médicale a pour but d'étudier les liens entre les facteurs d'enseignement et les résultats [...]. Les méthodes qualitatives [...] sont typiquement utilisées dans le cadre de simples études descriptives [...]. Elles sont de simples méthodes de collecte d'un type particulier de données » ${ }^{[35]}$. Les travaux récents continuent ainsi à évaluer la qualité de la recherche en éducation médicale sur la base exclusive des critères de rigueur des approches quantitatives ${ }^{[36,37]}$. Le fait que les pratiques de ce champ soient parfois considérées comme peu rigoureuses résulte par conséquent en partie d'une croyance selon laquelle seules les études expérimentales contrôlées - au demeurant peu nombreuses - remplissent les critères de qualité de la recherche scientifique ${ }^{[18]}$. De façon peu encourageante, Wilson écrivait récemment à ce sujet que « le pouvoir des études randomisées contrôlées et la médecine fondée sur les preuves ont tellement endoctriné les enseignants-cliniciens, que toute tentative de les changer conduira à un échec » ${ }^{[38]}$.

Le débat sur la place de la recherche qualitative en éducation médicale semble actuellement entretenu dans le contexte de la disciplinarisation du champ. Certains auteurs estiment ainsi que l'éducation médicale devait être appréhendée comme une science sociale et, de ce fait, en adopter les traditions de recherche ou, du moins, accepter d'élargir son spectre méthodologique ${ }^{[39-41]}$. Ils s'opposent aux chercheurs qui valorisent son identification aux disciplines biomédicales ${ }^{[42]}$.

\section{Synthèse : la «bataille » est-elle gagnée?}

Cette revue de la littérature nous a permis de retracer le développement de la recherche qualitative en pédagogie médicale, qui est étroitement comparable à l'essor de ces approches dans le domaine des sciences sociales et de l'éducation en général ${ }^{[8]}$. Toutefois, le débat sur leur légitimité en recherche en pédagogie médicale n'est de toute évidence pas clos, alors qu'en sciences sociales, «la «bataille» visant à en démontrer la pertinence, le caractère autonome et la scientificité face à la domination de 
l'optique quantitative a été gagnée il y a plus de 20 ans ${ }^{[4]}$.

Le constat que la proportion des travaux de recherche qualitative représentait, entre 2001 et 2005 , $30 \%$ des études publiées dans la «Revue des sciences de l'éducation » est en effet un témoin fort du fait que le débat qualitatif-quantitatif est dépassé en sciences de l'éducation ${ }^{[2]}$. Deschenaux et Laflamme considèrent ainsi que «le tournant des années quatre-vingt-dix semble être un moment où les tenants des méthodes qualitatives ont vu leurs capitaux prendre de la valeur, leur permettant de s'imposer dans le champ » ${ }^{[2]}$. Quant aux articles récents qui analysent les travaux de recherche publiés en pédagogie médicale, ils ne font pour la plupart pas mention des études qualitatives ${ }^{[36,37]}$. Certains auteurs focalisent explicitement d'emblée leur analyse sur les travaux de recherche expérimentale, considérant «qu'ils représentent le gold standard pour évaluer les conséquences des interventions pédagogiques en éducation et que leur analyse devrait être relativement simple, étant donné que les règles de l'expérimentation sont bien codifiées dans le domaine des sciences en général » ${ }^{[43]}$. Wilson évoque un problème de diffusion des études qualitatives dans le champ de l'éducation médicale, qu'il attribue au fait qu'elles apparaissent aux yeux des lecteurs comme de simples opinions - parfois exprimées dans un langage peu accessible - qui confèrent à ces travaux un caractère peu scientifique $^{[38]}$.

Lingard écrivait en 2007 que « heureusement, la recherche en éducation médicale a dépassé le débat (fatigant) quant à la question de savoir si la recherche qualitative est aussi «bonne » et « valable » que la recherche quantitative ${ }^{[20]}$. Au regard de la discussion qui précède, il semblerait pourtant que ce débat soit toujours d'actualité et que la «bataille» de la légitimité ne se soit pas encore gagnée. Nous rejoignons en cela l'avis récemment exprimé par Bunniss et Kelly, qui considèrent que « des débats méthodologiques ont toujours lieu dans le champ de la recherche en éducation médicale ${ }^{[16]}$. En outre, comme le souligne Britten, « une acceptation plus large ne signifie pas pour autant que les méthodes qualitatives sont bien comprises » ${ }^{[10]}$. C'est précisément à la façon dont les méthodes qualitatives et ses courants sont pratiqués dans le champ de la recherche en pédagogie médicale que nous allons nous intéresser dans la prochaine section.

\section{Pratique de la recherche qualitative et de ses courants en éducation médicale}

Nous souhaiterions d'emblée souligner que beaucoup de travaux publiés en éducation médicale sont peu explicites quant à la démarche de traitement des données et au courant auquel les chercheurs se réfèrent. Souvent, la discussion méthodologique évoque de façon évasive « une méthode de codage », sans que le lecteur bénéficie d'informations additionnelles ${ }^{[8]}$. Même lorsque la nature du courant est précisée, les auteurs donnent très peu de détails sur leur manière de procéder, ce qui ne permet pas de s'assurer qu'ils en ont compris les fondements et qu'ils en ont respecté les principes ${ }^{[19]}$.

Les approches qualitatives comme méthode de recherche

Dans un article de 1996 portant sur l'analyse des publications en éducation médicale, la recherche qualitative y était réduite à une méthode de recueil et de traitement des données ${ }^{[24]}$. Cette conception est un témoin fidèle de la façon dont ces approches sont appréhendées dans le champ de l'éducation médicale. Ce terme y est en effet avant tout utilisé pour désigner les outils de recueil et d'analyse des données auxquels le chercheur a recours ${ }^{[1]}$. Cette vision très réductrice est pourtant vivement critiquée, tant dans le champ des sciences humaines et sociales, que dans celui de l'éducation médicale. Ainsi, pour Guba et Lincoln, « les questions de méthode sont secondaires par rapport aux questions de paradigme, que nous définissons comme les croyances ou une vision du monde qui guide le 
chercheur, non seulement dans les choix méthodologiques, mais également ontologiques et épistémologiques » ${ }^{[9]}$. Pour Anadón, « la recherche qualitative en référence à des techniques particulières est trop restrictive, car elle doit davantage être associée à des postures épistémologiques et théoriques privilégiant d'une façon ou d'une autre l'expérience et les points de vue des acteurs sociaux ${ }^{[21]}$. Enfin, Lingard indique que «les outils eux-mêmes ne sont pas l'essence du paradigme qualitatif ${ }^{[20]}$. Dès la fin des années quatre-vingt, un article publié dans une revue d'éducation médicale mettait en garde les chercheurs à propos de cette conception simpliste et « amputatoire » de la démarche qualitative. Smith et Noblit y écrivaient que «il est incorrect de qualifier une étude "d'ethnographique », simplement parce qu'elle en utilise les techniques. [...] nous reconnaissons que la séparation des techniques de leur philosophie et de leur tradition est controversée » ${ }^{[17]}$. Quinze ans plus tard, Atkinson et Pugsley rappelaient à nouveau, dès l'introduction de leur article consacré au courant ethnographique en éducation médicale, que cette approche n'est pas une simple méthode de recueil et d'analyse des données ${ }^{[14]}$. En ce qui concerne la théorie ancrée, cette restriction des courants de la recherche qualitative à leur dimension méthodologique nous semble moins contestable, dans la mesure où la théorie ancrée peut aussi bien relever d'une approche globale de recherche, que d'une méthode d'analyse ${ }^{[21,44,45]}$.

Cette vision réductrice conduit à une forme de pratique de la recherche qualitative dans ce champ que nous qualifions « d'a-paradigmatique ».

Une pratique "a-paradigmatique » de la recherche

À l'opposé des chercheurs en sciences humaines et sociales, les chercheurs ancrés dans une tradition positiviste de la recherche font rarement mention de leur posture épistémologique et n'en sont parfois même pas conscients ${ }^{[8,46]}$. Or, le simple fait de déclarer utiliser une méthode qualitative ne permet pas toujours de rattacher la démarche de recueil et d'analyse à un courant de la recherche qualitative ou à une posture épistémologique ${ }^{[8]}$. Pourtant, «inscrire la recherche à l'intérieur d'une théorie, d'une discipline et d'une posture épistémologique est essentiel, si nous voulons construire des connaissances et ne pas simplement décrire des catégories thématiques » ${ }^{[20]}$. Par ailleurs, la déclaration d'intention épistémologique est «pertinent[e], voire incontournable, dans un processus de quête de sens » ${ }^{[47]}$. La connaissance n'a en effet de sens que lorsqu'elle est appréhendée dans un ensemble plus large de suppositions sur la nature de la réalité ${ }^{[16]}$, ce qui renvoie également à l'importance de la dimension ontologique de la démarche de recherche. L'analyse qualitative peut ainsi s'inscrire dans différents paradigmes qui servent de guide et qui ont des conséquences majeures sur le design de recherche et sur les choix méthodologiques ${ }^{[8,16]}$.

Cette pratique de recherche «a-paradigmatique » est entretenue par le fait qu'il n'est pas nécessaire de considérer les aspects épistémologiques et ontologiques pour publier un article dans les journaux d'éducation médicale ${ }^{[16]}$. Comme le soulignent les acteurs influents de la recherche dans ce champ, ce sont surtout les éléments relatifs à la méthode et aux résultats qui y sont valorisés ${ }^{[48]}$. Par ailleurs, les restrictions de longueur imposées par les éditeurs ne sont pas propices à ce type de discussion, et, d'une façon générale, aux considérations d'ordre théorique ${ }^{[46]}$.

\section{Des principes méthodologiques appliqués partiellement et sélectivement}

Beaucoup de travaux réalisés en éducation médicale ne respectent pas les principes de la recherche qualitative, décrits tant dans les manuels de référence en sciences humaines et sociales, que dans les articles publiés dans les journaux du champ ${ }^{[8]}$.

En ce qui concerne la théorie ancrée, un écueil fréquent consiste à appliquer uniquement les techniques d'analyse, sans se conformer aux principes fondamentaux de l'itération et de l'échantillonnage 
théorique ${ }^{[8]}$. Beaucoup de chercheurs reproduisent ainsi leurs habitudes héritées d'une tradition positiviste de la recherche, dans laquelle la phase de recueil et l'étape d'analyse des données sont cloisonnées l'une de l'autre ${ }^{[29]}$. Par ailleurs, les auteurs se contentent souvent d'identifier des « catégories », sans faire émerger de théorie ${ }^{[29]}$. Il s'agit là d'un problème majeur dans un contexte où les chercheurs en éducation médicale considèrent que ce champ doit impérativement renforcer ses fondements et sa production théoriques ${ }^{[33,48-51]}$. De surcroît, la présence de cas discordants n'est que rarement mentionnée, alors qu'ils sont un élément important de validité, dans la mesure où ils rendent compte de la complexité du phénomène étudié ${ }^{[8,52]}$. Enfin, il y a une forte résistance à adopter une démarche principalement inductive, qui constitue pourtant l'essence de la théorie ancrée, même si celle-ci comportera forcément, à un moment donné, une part de déduction $^{[52]}$. Cette tension entre l'émergence d'une théorie ("emergence») et le rattachement parfois forcé des données à un cadre théorique préexistant ( forcing ») est également très marquée dans le domaine des sciences humaines et sociales ${ }^{[53]}$. Les chercheurs en éducation médicale semblent partisans d'un processus d'analyse «dual». Il s'agit, en amont du codage, d'identifier des thèmes à partir de travaux antérieurs réalisés sur un sujet similaire, tout en restant sensible aux thèmes émergents ${ }^{[28]}$.

\section{Des critères de rigueur hérités du positivisme}

La focalisation sur la dimension méthodologique des approches qualitatives et l'ancrage positiviste des chercheurs en éducation médicale ont généré, au sein de cette communauté, une forte préoccupation pour les critères de rigueur. À ce sujet, Anadón observe qu'en sciences humaines et sociales, «la communauté des chercheurs ne compte pas un ensemble de critères bien établis et bien définis pour baliser les pratiques » ${ }^{[21]}$. Pour Mukamurera et al., «le travail d'analyse [en recherche qualitative] est encore souvent laissé dans l'ombre et ressemble davantage à une opération bricolée », ce qui est de nature à renforcer la méfiance des chercheurs quantitatifs vis-à-vis d'une démarche fréquemment perçue comme « subjective » ${ }^{[47]}$.

Le questionnement sur les critères de rigueur de la recherche qualitative n'est pas propre au champ de l'éducation médicale. Dans le domaine des sciences humaines et sociales, il fait également l'objet de nombreux débats ${ }^{[1,54,55]}$ qui s'inscrivent dans une perspective de «transparence » et de «systématisation des pratiques ${ }^{[47]}$.

En éducation médicale, les critères de qualité adoptés sont le plus souvent ceux des approches quantitatives - tels que la validité, la fiabilité et l'objectivité - ce qui génère des préoccupations majeures quant à la taille de l'échantillon et des efforts orientés vers la généralisation des résultats $^{[8,19,20,28,36]}$. Si Miles et Huberman ${ }^{[56]}$, ainsi que Mukamurera et al. ${ }^{[47]}$ utilisent également sans complexe le jargon quantitativiste, ils s'appliquent toutefois à expliquer en quoi le sens de l'échantillonnage, de la généralisabilité, de la validité ou encore de la fiabilité diffère de celui qu'on attribue traditionnellement à ces termes dans les approches quantitatives. Gohier considère ainsi qu'il serait plus juste de parler en recherche qualitative de «rigueur méthodologique » que de « validité scientifique » ${ }^{57]}$.

Lingard qualifie d' « hybridisation » cette pratique de la recherche qualitative selon les règles de la recherche quantitative ${ }^{[20]}$. L'auteur y voit un aspect positif, dans la mesure où cette démarche permet de parler le «langage» de la pédagogie médicale. Elle écrit à ce sujet : « si nous ne voulons pas parler dans le vide, nous devons être capables de raconter l'histoire de nos travaux qualitatifs de manière à ce que le public puisse «l'entendre » et s'engager avec nous ». Lingard en dénonce aussi les dérives qui limitent la possibilité d'exploiter pleinement la richesse de ces approches. Par exemple, la volonté d'inclure un nombre important de sujets conduit les chercheurs en éducation médicale à ne réaliser qu'une description de surface des phénomènes auxquels ils s'intéressent. Par ailleurs, la quête démesurée de généralisabilité les incite à mettre l'accent sur l'objectivité, 
les normes et le consensus, plutôt que sur les découvertes et sur les révélations inattendues ${ }^{[20]}$.

\section{Une sur-utilisation des entretiens semi-dirigés}

L'entretien - notamment dans le cadre des groupes de discussion focalisée (focus groups) - constitue l'outil de recueil de données qualitatives le plus populaire en éducation médicale ${ }^{[1]}$. Il est fréquemment semi-dirigé, c'est-à-dire, guidé par une série de questions ouvertes prédéterminées. Britten dénonce cet «accent trop prononcé sur l'utilisation des entretiens et des focus groups comme méthodes de collecte des données et le désintérêt relatif pour les méthodes d'observation, plus focalisées sur les actions que sur les perceptions ${ }^{[10]}$. Le choix privilégié de cet outil est probablement lié au fait qu'il possède un « rendement élevé » qui satisfait «l'impatience » caractérisant les chercheurs du champ ${ }^{[20]}$. Pourtant, d'autres méthodes de recueil ont fait l'objet d'une description approfondie dans les revues et les ouvrages d'éducation $^{[1,8,26,58]}$.

L'entretien constitue également un outil central de la recherche qualitative dans les autres champs des sciences humaines et sociales ${ }^{[8]}$. Anadón souligne toutefois que «le pluralisme méthodologique s'impose au nom de la diversité des objets [étudiés par ces dernières] et de la nécessité d'adapter les méthodes à leur spécificité » ${ }^{[21]}$.

\section{Une méthodologie de l'exploration?}

Les approches qualitatives sont parfois considérées dans le champ de l'éducation médicale comme servant uniquement à mener des études exploratoires - utiles à la formulation d'hypothèses de recherche ${ }^{[36]}$ - qui devraient obligatoirement être complétées par une démarche quantitative. Colliver écrit à ce sujet : «la recherche qualitative pourrait tout au plus être une source d'idées et d'hypothèses à propos de l'enseignement et de l'apprentissage ${ }^{[7]}$. La conception d'une finalité principalement exploratoire des approches qualitatives fut perceptible dès la fin des années quatre-vingt, comme en témoignent les travaux réalisés par Weinholtz ${ }^{[8]}$.

Elle est également discutée dans le domaine des sciences humaines et sociales. Trudel, Simard et Vonarx écrivent à ce sujet : " on comprendra que la recherche qualitative, souvent conduite dans le cadre d'un paradigme méthodologique empirico-inductif puisse être définie systématiquement comme une recherche exploratoire », dans laquelle il s'agit de « circonscrire un objet de recherche, définir de nouvelles pistes de recherche, choisir des avenues théoriques ou identifier une méthode appropriée à l'objet et à nos objectifs de recherche » ${ }^{[59]}$.

Il en résulte que l'usage de méthodes mixtes et l'appel à recourir à cette modalité de recueil et d'analyse - est de plus en plus marqué en recherche en éducation médicale ${ }^{[28,60]}$. Kennedy et Lingard soulignent ainsi les bénéfices de combiner des données obtenues lors d'entretiens ou d'observations à celles analysées statistiquement à partir des résultats de questionnaires ${ }^{[28]}$. L'intérêt pour cette mixité méthodologique a également émergé dans le domaine des sciences humaines et sociales, au cours des années quatre-vingt. Depuis, elle est à l'origine d'un débat quant à sa pertinence épistémologique ${ }^{[61,62]}$.

\section{Conclusion}

La pratique des approches qualitatives en éducation médicale s'inscrit essentiellement dans le cadre du paradigme positiviste ou post-positiviste, marqué par une ontologie réaliste et une épistémologie objectiviste. Fondamentalement, elle renvoie à des questionnements, une méfiance, des doutes, des préoccupations et des débats qu'a connus - et, dans une certaine mesure, que connait encore - la communauté des chercheurs en sciences humaines et sociales.

Le caractère contemporain des discussions sur la légitimité, la finalité, les modalités de pratique et les critères de scientificité de la recherche qualitative en éducation médicale nous semble toutefois plus prononcé dans ce champ que dans celui des sciences humaines et sociales. 
Nous identifions plusieurs facteurs qui pourraient en expliquer la raison :

- Les travaux des chercheurs en éducation médicale prennent place dans l'environnement des facultés de médecine, imprégné par la culture de la recherche en sciences fondamentales et cliniques, qui s'inscrit de façon marquée dans le paradigme positiviste ou post-positiviste ${ }^{[8,16,19]}$.

- Les chercheurs en éducation médicale sont également pour certains des praticiens et des enseignants en médecine ${ }^{[63]}$. Albert et al. écrivent à ce sujet qu' «étant donné que le modèle biomédical est celui qui domine au sein du champ de la recherche en éducation médicale, [...] les chercheurs ont difficilement d'autres choix, s'ils souhaitent obtenir la reconnaissance de leurs pairs, que de s'y conformer ${ }^{[48]}$.

- Beaucoup de chercheurs en éducation médicale ne possèdent pas les connaissances leur permettant de s'engager dans une démarche de recherche qualitative, méthodologiquement exigeante. Britten souligne à ce sujet le manque de sources d'informations succinctes et accessibles décrivant ces approches et leurs applications potentielles en éducation médicale ${ }^{[10]}$.

- Les thématiques de recherche sont dans ce champ dominées par les questions d'évaluation, appréhendées traditionnellement grâce à la mesure ${ }^{[37,40]}$.

Un ressort de la nécessité et de la légitimité d'un élargissement du spectre méthodologique des chercheurs en éducation médicale aux approches qualitatives pourrait reposer sur la diversification des thématiques de recherche et d'enseignement, que connait ce champ depuis plusieurs années. Les chercheurs portent en effet un intérêt croissant aux questions de professionnalisation, de communication ou encore de collaboration interprofessionnelle ${ }^{[25]}$. La complexité de ces sujets rend improbable et incertaine leur étude à l'aide des outils traditionnels de la recherche quantitative. Elle légitime ainsi pleinement la place des approches qualitatives dans le champ disciplinaire de l'éducation médicale et la pertinence de la doctrine du «best-fit ».

\section{Contributions}

Les deux auteurs ont conjointement mené des discussions sur le contenu et la structuration de l'article. Thierry Pelaccia a rédigé une première version qui a fait l'objet d'une révision par Pierre Paillé. La version finale du manuscrit a été approuvée par les deux auteurs.

\section{Références}

1. Lingard L, Kennedy TJ. Qualitative research methods in medical education. In: Swanwick $\mathrm{T}$ (eds.). Understanding medical education: evidence, theory and practice. Londres: Wiley-Blackwell, 2010:323-5.

2. Deschenaux F, Laflamme C. Analyse du champ de la recherche en sciences de l'éducation au regard des méthodes employées : la bataille est-elle vraiment gagnée pour le qualitatif ? Recherches Qualitatives 2007;27:5-27.

3. Paillé P. La recherche qualitative... sans gêne et sans regrets. Recherche en soins infirmiers 1997;50:60-4.

4. Pelaccia T, Paillé P. Les approches qualitatives : une invitation à l'innovation et à la découverte dans le champ de la recherche en pédagogie des sciences de la santé. Pédagogie Médicale 2010;10:293-304.

5. Irby DM. Shifting paradigms of research in medical education. Acad Med 1990;65:622-3.

6. Norman G. On science, stories, quality and quantity. Adv Health Sci Educ Theory Pract 1998;3:77-80.

7. Colliver JA. Constructivism with a dose of pragmatism: a cure for what ails educational research. Adv Health Sci Educ Theory Pract 1999;4:187-90.

8. Harris IB. Qualitative methods. In: Norman GR, van der Vleuten CP et Newble DI (eds.). International handbook of research in medical education. Boston: Kluwer Academic, 2002:45-93.

9. Denzin N, Lincoln Y. Introduction: the discipline and practice of qualitative research. In: Denzin $\mathrm{N}$ et Lincoln $\mathrm{Y}$ (eds). Handbook of qualitative research. Thousand Oaks: Sage Publications, 2005:1-32.

10. Britten N. Making sense of qualitative research: a new series. Med Educ 2005;39:5-6.

11. Pollak O. [Commentaires sur l'ouvrage de Merton et al.]. The Annals of the American Academy of Political and Social Science 1960;328:207.

12. Laqueur T. Boys in white: student culture in medical school. BMJ 2002;325:721. 
13. Forquin JF. Les sociologues de l'éducation américains et britanniques. Bruxelles : De Boeck Université, 1997.

14. Atkinson P, Pugsley L. Making sense of ethnography and medical education. Med Educ 2005;39:228-34.

15. Wessely S. Making doctors: an institutional apprenticeship. BMJ 1998;316:713.

16. Bunniss S, Kelly D. Research paradigms in medical education research. Med Educ 2010;44:358-66.

17. Smith A, Noblit G. The idea of qualitative research in medical education. Teach Learn Med 1989;1:101-8.

18. Stacy R, Spencer $\mathrm{J}$ Assessing the evidence in qualitative medical education research. Med Educ 2000;34:498-500.

19. Harris IB. What does «The discovery of grounded theory » have to say to medical education? Adv Health Sci Educ Theory Pract 2003;8:49-61.

20. Lingard L. Qualitative research in the RIME community: critical reflections and future directions. Acad Med 2007;82:129-130.

21. Anadón M. La recherche dite «qualitative»: de la dynamique de son évolution aux acquis indéniables et aux questionnements présents. Recherches Qualitatives 2006;26:5-31.

22. Bordage G. La recherche en pédagogie médicale en Amérique du Nord : tour d'horizon et perspectives. Pédagogie Médicale 2000;1:9-12.

23. Collins J. Medical education research: challenges and opportunities. Radiology 2006;240:639-47.

24. Dimitroff A, Davis W. Content analysis of research in undergraduate medical education. Acad Med 1996;71:60-7.

25. Regehr G. Trends in medical education research. Acad Med 2004;79:939-47.

26. Pope C. Conducting ethnography in medical settings. Med Educ 2005;39:1180-7.

27. Bélisle R. Pratiques ethnographiques dans des sociétés lettrées : l'entrée sur le terrain et la recherche impliquée en milieux communautaires. Recherches Qualitatives 2001;22:55-71.

28. Kennedy TJ, Lingard LA. Making sense of grounded theory in medical education. Med Educ 2006;40:101-8.

29. Heath H, Cowley S. Developing a grounded theory approach: a comparison of Glaser and Strauss. Int J Nurs Stud 2004;41:141-50.

30. Bryant A, Charmaz K. The Sage handbook of grounded theory. Thousand Oaks: Sage Publications, 2007.

31. Yin RK. Case study research: design and methods. Thousand Oaks: Sage Publications, 2008
32. Meyor C. La phénoménologie dans la méthode scientifique et le problème de la subjectivité. Recherches Qualitatives 2005;25:25-42.

33. Wartman SA. Research in medical education: the challenge for the next decade. Acad Med 1994;69:608-14.

34. Wood D, Bligh J. Medical education comes of age. Med Educ 2000;34:82-3.

35. Colliver JA, Verhulst SJ. Medical research and qualitative methods: a rational approach. Acad Med 1996;71:211.

36. Baernstein A, Liss HK, Carney PA, Elmore JG. Trends in study methods used in undergraduate medical education research, 1969-2007. JAMA 2007;298:1038-45.

37. Todres M, Stephenson A, Jones R. Medical education research remains the poor relation. BMJ 2007;335:333-5.

38. Wilson I. Qualitative research in medical education. Med Educ 2010;44:942.

39. Dornan T, Peile E, Spencer J. On «evidence ». Med Educ 2008;42:232-3.

40. Kuper A, Reeves S, Albert M, Hodges BD. Assessment: do we need to broaden our methodological horizons? Med Educ 2007;41:1121-3.

41. Monrouxe LV, Rees CE. Picking up the gauntlet: constructing medical education as a social science. Med Educ 2009;43:196-8.

42. Bligh J, Brice J. What is the value of good medical education research? Med Educ 2008;42:652-3.

43. Cook DA, Beckman TJ, Bordage G. A systematic review of titles and abstracts of experimental studies in medical education: many informative elements missing. Med Educ 2007;41:1074-81.

44. Paillé P. L'analyse par théorisation ancrée. Cahiers de Recherche Sociologique 1994;23:147-81.

45. Paillé $P$. Une «enquête de théorisation ancrée »: les racines et les innovations de l'approche méthodologique de Glaser et Strauss. In : Glaser BG et Strauss AL (eds). La découverte de la théorie ancrée. Paris : Armand Colin, 2010:23-77.

46. Rees CE, Monrouxe LV. Theory in medical education research: how do we get there? Med Educ 2010;44:334-9.

47. Mukamurera J, Lacourse F, Couturier Y. Des avancées en analyse qualitative : pour une transparence et une systématisation des pratiques. Recherches Qualitatives 2006;26:110-8.

48. Albert M, Hodges B, Regehr G. La recherche en éducation médicale : entre le service et la science. Pédagogie Médicale 2006;7:73-81.

49. Colliver JA. Educational theory and medical education practice: a cautionary note for medical school faculty. Acad Med 2002;77:1217-20. 
50. Bligh J. Nothing is but what is not. Med Educ 2003;37:184-5.

51. Prideaux D, Spencer J. On theory in medical education. Med Educ 2000;34:888-9.

52. Guillemette F. L'approche de la Grounded Theory: pour innover ? Recherches Qualitatives 2006;26:32-50.

53. Kelle U. «Emergence» vs. «forcing » of empirical data? A crucial problem of « grounded theory » reconsidered. Forum: Qualitative Social Research 2005;6.

54. Drapeau M. Les critères de scientificité en recherche qualitative. Pratiques Psychologiques 2004;10:79-86.

55. Drapeau M, Letendre R. Quelques propositions inspirées de la psychanalyse pour augmenter la rigueur en recherche qualitative. Recherches Qualitatives 2001;22:73-92.

56. Miles MB, Huberman AM. Analyse des données qualitatives. Bruxelles : De Boeck Université, 2003.

57. Gohier C. De la démarcation entre critères d'ordre scientifique et d'ordre éthique en recherche interprétative. Recherches Qualitatives 2004;24:3-17.
58. Bleakley A. Stories as data, data as stories: making sense of narrative inquiry in clinical education. Med Educ 2005;39:534-40.

59. Trudel L, Simard C, Vonarx N. La recherche qualitative est-elle nécessairement exploratoire ? Recherches Qualitatives 2007;5:38-45.

60. Dicicco-Bloom B, Crabtree BF. The qualitative research interview. Med Educ 2006;40:314-21.

61. Paillé P. Lumières et flammes autour de ma petite histoire de la recherche qualitative. Recherches Qualitatives 2006;26:139-53.

62. Pinard R, Potvin P, Rousseau R. Le choix d'une approche méthodologique mixte de recherche en éducation. Recherches Qualitatives 2004;24:58-80.

63. Albert M. Understanding the debate on medical education research: a sociological perspective. Acad Med 2004;79:948-54.

Correspondance et offprints : Thierry Pelaccia, SAMU 67, CESU 67, Pôle logistique des Hôpitaux universitaires de Strasbourg, 70 rue de l'Engelbreit, 67200 Strasbourg, France.

Mailto : thierry.pelaccia@wanadoo.fr 